\section{Running on empty}

the changes
detected in
mouse models
of cancer
were evident
in patients
with cancer-
associated
cachexia

The search for effective treatments for cachexia, a paraneoplastic syndrome that leads to the loss of white adipose tissue (WAT) and skeletal muscle, has been hampered by a lack of understanding of the molecular basis of this syndrome.

Both Erwin Wagner and colleagues and Bruce Spiegelman and colleagues found that the generation of brown adipose cells in WAT (also known as beige or brite cells) was an early change that occurred before the clinical appearance of cachexia in mice. Beige cells express uncoupling protein 1 (UCP1), a mitochondrial protein that switches mitochondrial respiration from ATP generation to thermogenesis, indicating an alteration from energy storage in WAT to heat generation and the radiation of energy by brown adipose tissue (BAT).

Wagner and colleagues showed that the increased formation of beige cells occurred in several different mouse models of cancer. Energy

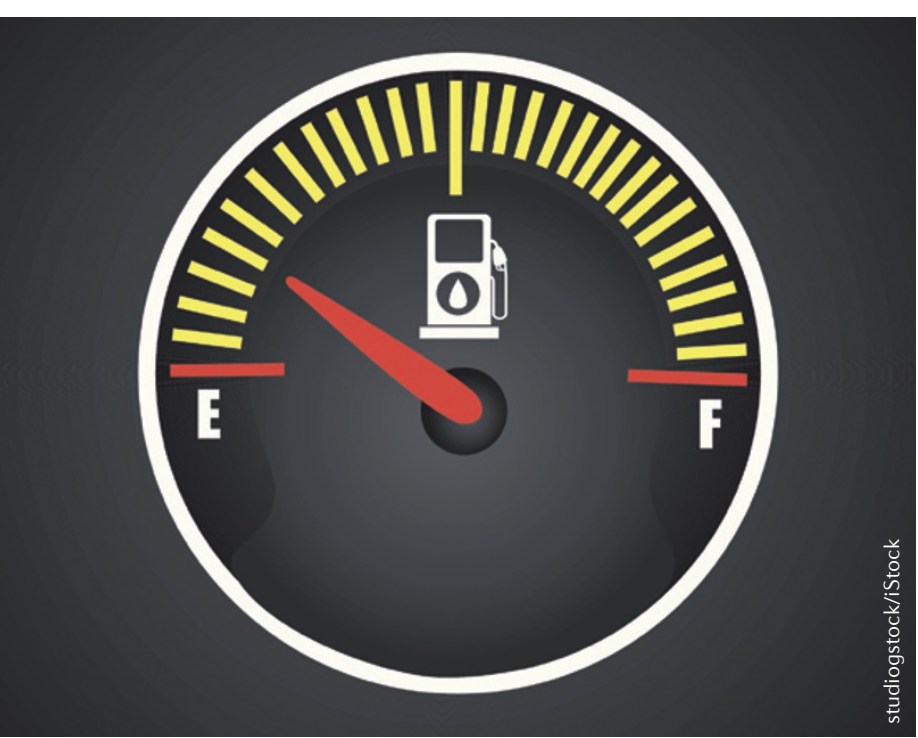

expenditure and concomitant lipolysis in WAT in these mice increased as the tumours progressed, despite the mice having decreased physical activity. Consistent with the hypothesis that inflammation is involved in cachexia, they found that interleukin-6 (IL-6) expression is increased in cachectic mice. Consistent with this, transplantation of IL-6-expressing tumour cells in mice resulted in substantial cachexia compared with mice transplanted with tumour cells that do not express IL-6. Moreover, the use of an IL-6-blocking antibody or transplantation of melanoma cells into IL-6 receptor-deficient mice reduced but did not fully suppress cachexia. These authors also found that the activity of $\beta$-adrenergic-responsive neurons, which are known to induce beige cells in cold conditions, is also involved in the induction of cachexia in mice. The blockade of $\beta 3$-adrenergic receptors using antagonists or of inflammation using a non-steroidal anti-inflammatory drug significantly reduced the onset of cachexia in mice, and this was associated with reduced expression levels of UCP1 in beige cells.

Spiegelman and colleagues used mouse models of Lewis lung carcinoma (LLC) to examine whether tumour-derived factors were involved in the increased expression of genes involved in thermogenesis, glucose transport, $\beta$-oxidation and lipolysis that occur in these mice. Fractionation of tumour-conditioned media indicated that tumours produce a thermogenic macromolecule and further studies narrowed this down to several members of the epidermal growth factor (EGF) family and parathyroidhormone-related protein (PTHRP).
The use of a protein kinase inhibitor to inhibit EGF receptors indicated that PTHRP was the more likely candidate. PTHRP signals through the PTH receptor, a $G$ protein-coupled receptor (GPCR) that activates the cyclic-AMP-dependent protein kinase A (PKA) pathway, as do $\beta$-adrenergic receptors. PKA inhibitors indicated that activation of PKA downstream of GPCRs was required for the induction of thermogenic gene expression in primary white and brown adipocytes in culture. Moreover, a neutralizing antibody specific for PTHRP significantly inhibited expression of $U c p 1$ in adipocytes exposed to tumour-conditioned media in vitro and prevented WAT and skeletal muscle loss in LLC mice. The injection of PTHRP into healthy mice showed that this protein does not directly induce skeletal muscle atrophy, but when injected into LLC mice, it increases cachexia-associated fat and muscle wasting. These findings indicate that although PTHRP is required for skeletal muscle wasting and loss of function, other tumour-derived factors are likely to also be important.

Both groups also showed that the changes detected in mouse models of cancer were evident in patients with cancer-associated cachexia. Thus, these papers indicate potential new treatment strategies in the prevention and treatment of cachexia.

Nicola McCarthy, Horizon Discovery Group Inc

The author declares no competing financial interests.

ORIGINAL RESEARCH PAPERS Petruzzelli, M. et al. A switch from white to brown fat increases energy expenditure in cancer-associated cachexia. Cell Metab. http://dx.doi.org/10.1016/j. cmet.2014.06.011 (2014)|Kir, S. et al. Tumourderived PTH-related protein triggers adipose tissue browning and cancer cachexia. Nature http://dx.doi.org/10.1038/nature13528 (2014) 